\title{
FINGERTIP FORCE ESTIMATION FROM FOREARM MUSCLE ELECTRICAL ACTIVITY
}

\author{
Pu Liu ${ }^{*}$, Francois Martel $^{\dagger}$, Denis Rancourt ${ }^{\dagger}$, Edward A. Clancy ${ }^{*}$ and D. Richard Brown III \\ *Worcester Polytechnic Institute, Worcester, MA, USA, \{puliu, ted, drb \}@wpi.edu \\ ${ }^{\dagger}$ University of Sherbrooke, Sherbrooke, QC, Canada, \{Francois.Martel2, \\ Denis.Rancourt\}@USherbrooke.ca
}

\begin{abstract}
Existing commercial hand prostheses can be controlled from the electrical activity (electromyogram or EMG) of remnant muscle tissue within the forearm, but are limited in function to one degree of freedom of proportional control. In a pilot study ( $N=3$ subjects), we used least squares estimation to identify a model between forearm electrical activity recorded by high-resolution (64 channel) electrode arrays (applied over the flexor and, separately, extensor muscles of the forearm) to force in the four fingertips. Average errors ranged from 4.21 to $10.20 \% \mathrm{MVC}_{\mathrm{F}}$ (flexion maximum voluntary contraction), depending on the muscle contraction task performed, number of EMG electrodes in the model and the electrode montage selected. Results suggest that, at least for intact subjects, 2-4 degrees of freedom of proportional control are available from the EMG signals of the forearm.
\end{abstract}

Index Terms - EMG signal processing, biomedical signal processing, EMG-force, electromyography

\section{INTRODUCTION}

Classic myoelectric control of a hand prosthesis provides, at most, one degree of freedom of proportional control from the electromyogram (EMG) of one extension electrode and one flexion electrode, each placed on the skin over the remnant muscle tissue of the forearm $[1,2]$. Amputees desire improved control capabilities, particularly an increase in the number of degrees of freedom [1,2]. One approach to increased control is multifunction selection in which classification analysis is used to relate features derived from forearm EMG to various hand/wrist functions [3-9]. Classification accuracies above 95\% have been achieved, with higher accuracies found when more electrodes are used, fewer functions are selected and/or longer EMG signal durations are observed. Some studies have concentrated on classification of individual finger movements [10-14]. This approach can increase amputee function, but does not provide the desired proportional control.

Supported by U.S. Army under USAMRAA grant W81XWH-08-1-0422.
Some recent effort [14-17] has concentrated on the goal of providing proportional finger control via EMGbased estimation of finger joint angles or forces. Force estimation may be preferable, as it is likely to be less influenced by external forces that interact with the hand. However, many questions remain, particularly with respect to the number of electrodes required and how their signal should be acquired and processed. In particular, the muscles of the forearm are small in cross section and packed tightly beside each other, making it difficult to sense their activities independently. Over the past few years, high resolution spatial filtering of EMG array signals has been used to localize the electrical potentials of small muscle tissue volumes [18-20]. We hypothesized that such systems would be useful in separating the source electrical activity of distinct hand muscles within the forearm, facilitating more accurate EMG-force identification.

This paper presents the methods and results of a pilot study ( $N=3$ subjects) in which commercial high-resolution (64 channel) electrode arrays were used to measure EMG signals from the extensor and flexor muscles of the forearm while recording fingertip flexion-extension forces during constant-posture contractions. The goal of the study was to investigate and compare the performance of various EMG spatial filters ("montages") in terms of their ability to identify an EMG-force relationship for the fingertips. Our results showed average errors ranged from 4.21 to 10.20 $\% \mathrm{MVC}_{\mathrm{F}}$, depending on the muscle contraction task performed, number of EMG electrodes in the model and the electrode montage selected. Our results also suggest that, at least for intact subjects, 2-4 degrees of freedom of proportional control are available from the forearm EMG.

\section{METHODS}

\subsection{Experimental Apparatus}

The arm restraint device, shown in Fig. 1, was used to record constant-posture finger flexion-extension. The subject sat along the table edge with their elbow forming a $90^{\circ}$ angle. The height of the elbow rest plate was adjusted for each finger to keep the long axis of the forearm parallel to the table. After donning a glove, the palm of the hand 


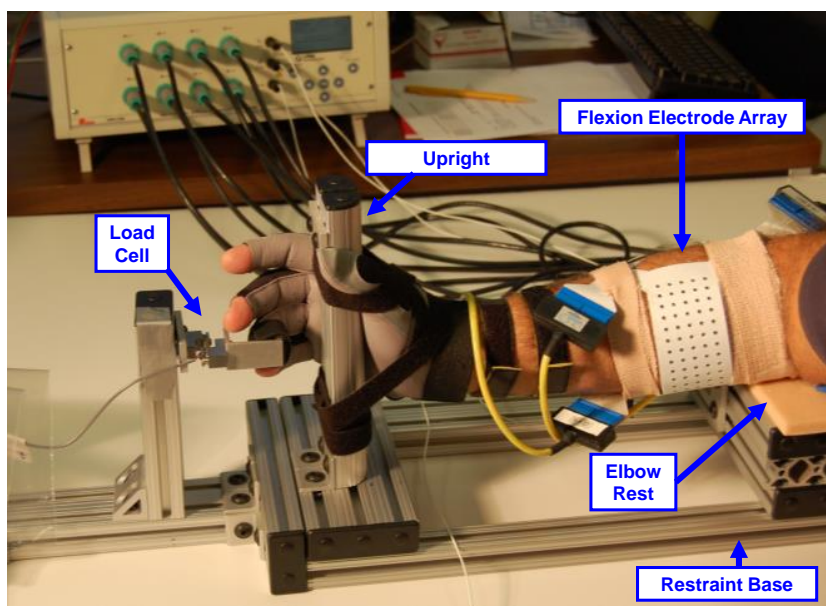

Fig. 1. Photograph of hand/arm secured into the finger restraint. Electrode arrays are mounted over the medial (flexion array) and lateral (extension array - not visible) aspects of the forearm.

was secured at the front of the restraint to an upright via Velcro. The hand was oriented with the thumb directed upwards and the four remaining digits passively curled beyond the upright. The height of the hand was adjusted to align the distal phalange of any one digits with the load beam. A phalange was secured to the load beam by a tightly-wrapped Velcro strip. Load on this beam was measured with a one degree of freedom load cell and amplifier (models LCL-040 and DMD-465WB, respectively; Omega Engineering, Inc., Stamford, CT, USA). The cut-off frequency of the amplifier lowpass filter was $9.4 \mathrm{~Hz}$ (second-order, Bessel). Measurement was only made on one digit at a time.

The skin over the circumference of the proximal right forearm was scrubbed with an alcohol wipe. Two, commercial 64-channel monopolar electrode arrays were applied (ELSCH064R3S Adhesive Electrode Arrays, EMGUSB Amplifier; OT Bioeletronica, Torino, Italy). Each array was a rectangular, $13 \times 5$ matrix of electrodes (with one corner electrode omitted), utilizing $2 \mathrm{~mm}$ diameter gel-filled electrodes separated by $8 \mathrm{~mm}$ center-to-center. The long axis of the "flexion" array was oriented along the circumference of the right forearm, centered on the mid-line of the medial aspect of the forearm. The second "extension" array was secured with the long axis oriented along the right forearm circumference, centered on the mid-line of the lateral aspect of the forearm. The eight extension electrodes located furthest from the base of the finger restraint along the most proximal electrode column were not used, leaving 56 electrodes. A gap of $3.5-7 \mathrm{~cm}$ existed between the superior and inferior edges of the two electrode arrays. The proximal edge of each EMG array was located three fingers width from the olecranon process [21]. A reference electrode was applied to the left wrist and a power-line attenuation circuit ("driven-right-leg") was applied to the right arm. Each electrode channel had a passband from 10$750 \mathrm{~Hz}, \mathrm{CMRR}$ greater than $104 \mathrm{~dB}$ at the power line frequency, input impedance greater than $10^{14} \Omega$, and input referred noise $<1 \mu \mathrm{V}$ RMS. EMG data were sampled at $2048 \mathrm{~Hz}$ with 12-bit resolution.

A PC was used to collect the finger flexion-extension load cell data $(128 \mathrm{~Hz}, 16$ bits; synchronized offline with the EMG data) and as a subject display. Its 18 inch monitor was placed approximately one meter in front of the subject. A custom LabView interface displayed a vertical line on the screen that moved horizontally with the subject's extensionflexion force. A fixed or dynamic target could also be displayed on the screen.

\subsection{Experimental Methods}

The New England IRB approved and supervised the human studies. Three subjects each completed one experiment. Subjects had no known neuromuscular deficits of their right hand, arm or shoulder. After signing written informed consent, subjects were fitted into the hand restraint device. Each subject performed separate maximum flexion, then extension trials for each of the four digits, repeated twice. The average flexion plateau for each digit and the average extension plateau for each digit were used as the respective maximum voluntary contraction (MVC) values. Subsequent contractions were scaled to the MVC of the respective digit. The EMG electrode arrays were then secured (see above).

Subjects next performed five-second constant-force contractions. Two such recordings were made for $30 \%$ MVC flexion and, separately, 30\% MVC extension, for each digit. Subjects lastly performed a series of slowly force-varying (ramp) tracking tasks. The LabView display of extension-flexion force was scaled over the range from $30 \%$ MVC extension to 30\% MVC flexion. A target signal began at the force level half-way between these two extremes (this level was not equivalent to zero force, since extension and flexion MVCs are not equal), advanced to $30 \%$ extension, continued to $30 \%$ flexion, returned to $30 \%$ extension, and ended back at the half-way force. Tracking lasted for 30 seconds, with all target movement at a constant speed. Four tracking tasks were completed per digit.

\subsection{Methods of Analysis}

Data Preprocessing: Data analysis was performed off-line using MATLAB. The sampled EMG data were bandpass filtered $(15-700 \mathrm{~Hz})$ using a fourth-order Butterworth filter, and second-order notch filters at the power line frequency and all harmonics. Filtering was applied in the forward, then reverse time directions to achieve zero phase. Each data recording was plotted and reviewed. Channels with anomalous data (e.g., obviously corrupted by excessive power line noise or motion artifact) were avoided from further use. Regardless, all desired electrode configurations were achieved. The finger force data were upsampled to the same rate as the EMG data (2048 Hz), time-aligned to the 


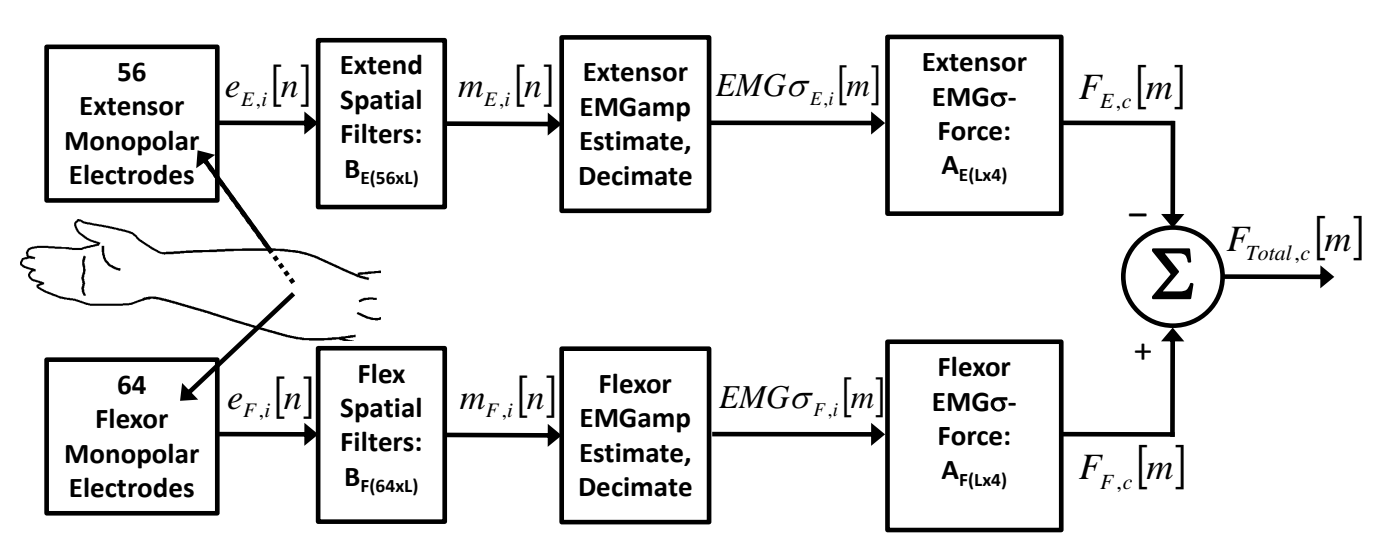

Fig. 2. EMG-force model. Extension and flexion monopolar arrays are spatially filtered into $L$ signals, each

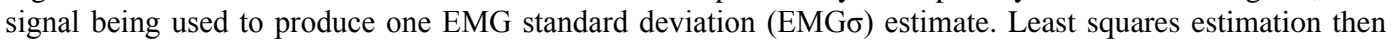
relates the EMGo's to force of the four fingertips (indexed by $c$ ). The " $B$ " matrices hold the coefficients of the spatial filters; the " $A$ " matrices hold the coefficients relating EMG $\sigma$ to force. two test crossvalidations was expressed in percent MVC flexion $\left(\% \mathrm{MVC}_{\mathrm{F}}\right)$, relative to each respective digit.

For the slowly

force-varying (ramp) contractions, the first and last five seconds of each 30 second tracking trial were discarded, leaving one complete contraction cycle of duration 20 seconds per digit. Four sequential tracking

EMG data and scaled to its respective flexion MVC value. The fingertip force for inactive fingers was set to zero.

EMG-Force Processing: The EMG-force model is shown in Fig. 2. Numerous classic spatial filters with known (pre-selected) spatial filter coefficients were investigated. The preprocessed extensor/flexor signal sets $\left(e_{E, i}[n]\right.$, $e_{F, i}[n]$, where $i$ indexes the spatial channels and $n$ indexes time) were spatially filtered to produce $L$ extensor/flexor channels $\left(m_{E, i}[n], m_{F, i}[n]\right)$. A spatial filter is a memory-less weighted sum of the monopolar potentials. The EMG standard deviation (EMG amplitude estimate) of each channel was computed by rectifying each channel and then decimating to $10.24 \mathrm{~Hz}$. After decimating, the signal was further lowpass filtered (cut-off frequency of $1 \mathrm{~Hz}$, fourthorder Butterworth filter applied in the forward, then reverse time directions), producing signals $E M G \sigma_{E, i}[m]$ and $E M G \sigma_{F, i}[m]$, where $m$ indexes time at the reduced rate. This reduced rate is appropriate as it is approximately ten times that of the force signal being estimated [22, 23].

For the constant-force recordings, distinct five second flexion and extension recordings from each finger were concatenated, forming a 40 second data set (4 fingers x 10 seconds per finger). A fit coefficient was multiplied by each of the $L$ extension EMG $\sigma$ 's to estimate each of the four digit extension force contributions (total of $4 L$ coefficients). Another $4 L$ coefficients were similarly required to estimate flexion force contributions. Their difference was the estimate of total force for each finger. Linear least squares was used to estimate the fit coefficients from a 40 second data set. Since there were two constant-force recordings per finger per flexion/extension contraction, two such sets were available per subject. One set was used for coefficient training and the second for performance testing, with full leave-one-out cross-validation. The average error from the recordings, representing data from each of the four digits, were concatenated to form an 80 second data set. Linear least squares was again used to estimate fit coefficients (same method as described above) from an 80 second tracking set. Four tracking data sets were available per subject. Three data sets were used for coefficient training and the fourth for performance testing, with full leave-oneout cross-validation. The average error from the four crossvalidations was expressed in $\% \mathrm{MVC}_{\mathrm{F}}$, relative to each respective digit.

For each of the constant-force and ramp contraction data sets, each extension/flexion EMG array contained 13 rows of electrodes. An $L=13$ channel monopolar spatial filter (montage) was formed by choosing one of the central electrodes in each row. Then, alternate rows were selected to form an $L=7$ channel monopolar spatial filter. By skipping increasingly more rows, filters were formed for $L=5$ and 4 channels. Next, these four row selections were repeated, utilizing additional adjacent columns to form bipolar and linear double difference (LDD) filters [20]. Note that these filters were formed along the presumed direction of action potential propagation. Lastly, normal double difference (NDD) filters were formed. Because of the additional rows required to form NDD filters, the selected channel sizes were $L=11,6$ and 4 . Thus, a total of 15 classic spatial filters were investigated.

\section{RESULTS}

Fig. 3 shows sample results from the constant-force trials using a 5-channel, bipolar electrode montage. Table I shows the complete summary results for all montages and number of EMG channels studied. Fig. 4 shows sample results from the slowly force-varying (ramp) trials using a 13-channel monopolar montage. Table II shows the complete summary results. Taken together, the results suggest that the montages known to be more spatially selective (LDD and 


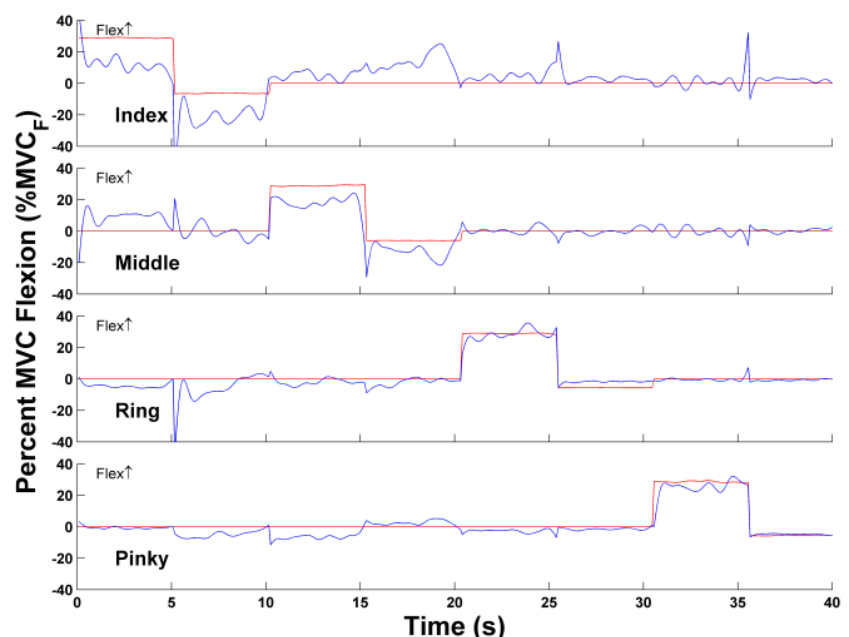

Fig. 3. Constant-force trial sample EMG-force test results of estimated (jagged blue line) and actual (solid red line) force vs. time using $L=5$ bipolar montage. Subject WZ04, trials 01-08.

TABLE I

Constant-Force TRIAL Average RMS Test ERror Results $\left(\% \mathrm{MVC}_{\mathrm{F}}\right)$

\begin{tabular}{c|c|c|c|c} 
EMG Channels & \multicolumn{4}{|c}{ Spatial Filter } \\
\cline { 4 - 5 }$(L)$ & Mono & Bipolar & LDD & NDD \\
\hline \hline 13 (11 for NDD) & 9.25 & 8.03 & 10.02 & 10.03 \\
7 (6 for NDD) & 8.95 & 8.99 & 8.64 & 10.20 \\
5 & 8.34 & 7.22 & 10.31 & - \\
4 & 7.82 & 8.43 & 8.17 & 8.82 \\
\hline \hline
\end{tabular}

NDD) did not produce lower EMG-force estimation errors; in fact, their errors were generally higher. There was also not a strong trend for lower errors as the number of EMG channels was increased. The constant-force results seem to show higher average errors overall, perhaps due to the small duration of signal (5 seconds) available for training [24]. Given the small number of subjects, statistical comparisons were not pursued.

\section{DISCUSSION AND CONCLUSIONS}

The EMG-force errors found in this study (ranging from 4.21 to $10.20 \% \mathrm{MVC}_{\mathrm{F}}$ ) are similar to errors found in studies of other joints (c.f., [24]). This outcome is significant, since there is currently no consensus within the literature that multiple degrees of freedom of proportional control are available - at least in intact subjects - to relate forearm electrical activity to fingertip forces. Further, these results suggest that there may be no obvious advantage to highresolution (and high channel count) electrode arrays and spatial montages. Such arrays are thought to reduce EMG crosstalk (undesired recording of more distant muscles away from the recording site). Crosstalk is thought to confound EMG-force identification, although blind source separation techniques have been attempted to resolve this problem

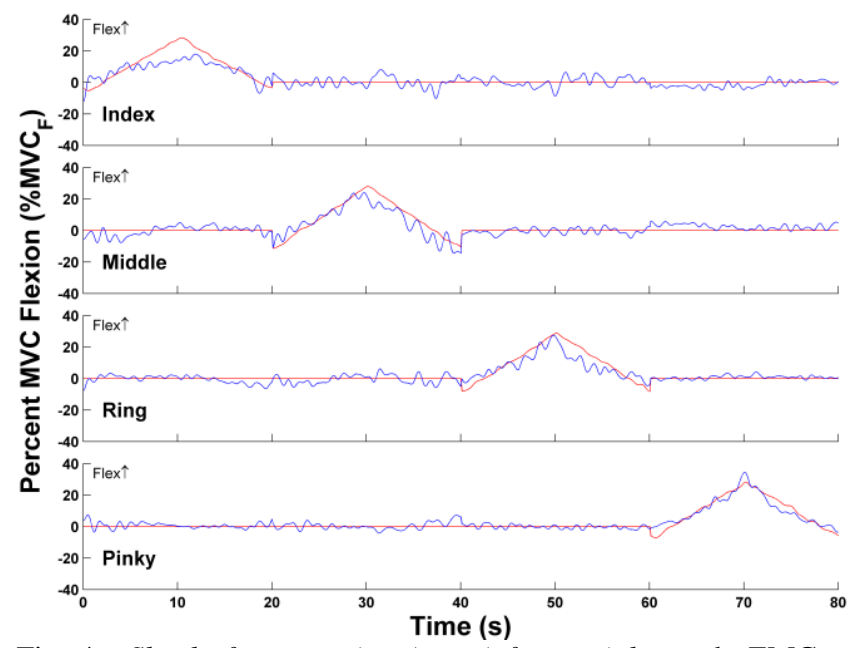

Fig. 4. Slowly force-varying (ramp) force trial sample EMGforce test results of estimated (jagged blue line) and actual (solid red line) force vs. time using $L=13$ monopolar montage. Subject WZ09, trials 26, 27, 36, 37 .

TABLE II

SLOWLY ForCE-VARYING (RAMP) TRIAL AVERAGE RMS TEST ERROR RESULTS $\left(\% \mathrm{MVC}_{\mathrm{F}}\right)$

\begin{tabular}{c|c|c|c|c} 
EMG Channels & \multicolumn{4}{|c}{ Spatial Filter } \\
$(L)$ & Mono & Bipolar & LDD & NDD \\
\hline \hline $13(11$ for NDD) & 4.41 & 5.49 & 5.97 & 5.51 \\
7 (6 for NDD) & 4.51 & 5.68 & 5.73 & 5.58 \\
5 & 4.69 & 5.37 & 5.91 & - \\
4 & 4.84 & 5.45 & 5.99 & 5.51 \\
\hline \hline
\end{tabular}

[25]. If high resolution arrays provide little or no advantage, then standard electrode hardware might be used instead. Such hardware is simpler, less expensive and more readily available. In fact, existing commercial high resolution arrays are not suitable (or designed) for use in commercial prostheses.

The sample size used in this study was small and primarily intended as part of a pilot study. While the results are encouraging, a number of limitations exist, including: the lack of dynamics in the contraction forces, the limitation of constant-posture contractions, the omission of models that incorporate the thumb (necessary for many hand actions/grips) and the limited model forms studied.

In summary, EMG signals were acquired from the extensor and flexor muscles of the forearm during constantposture contractions and related to the force produced in the four fingers (index, middle, ring and pinky). Various conventional electrode montages and number of EMG channels were considered. Over a range of contraction forces spanning $30 \% \mathrm{MVC}$ extension to $30 \% \mathrm{MVC}$ flexion, RMS EMG-force error ranged from 4.21-10.20\% $\mathrm{MVC}_{\mathrm{F}}$, depending on the montage and number of channels. Results were encouraging for finger EMG-force applications in prosthesis control. 


\section{REFERENCES}

[1] P. A. Parker, K. Englehart and B. Hudgins, "Myoelectric signal processing for control of powered limb prostheses," $J$. Electromyo. Kinesiol., vol. 16, pp.541-548, 2006.

[2] D. Atkins, D. C. Y. Heard and W. H. Donovan, "Epidemiologic overview of individuals with upper-limb loss and their reported research priorities," J. Prosthet. Orthot., vol. 8, pp. 2-11, 1996.

[3] B. Hudgins, P. A. Parker, R. N. Scott, "A New Strategy for Multifunction Myoelectric Control," IEEE Trans. Biomed. Eng., vol. 40, pp.82-94, 1993.

[4] K. A. Farry, I. D. Walker and R. G. Baraniuk, "Myoelectric Teleoperation of a Complex Robotic Hand," IEEE Trans. Robot. Automat., vol. 12, pp. 775-788, 1996.

[5] K. Englehart, B. Hudgins and P. A. Parker, "A WaveletBased Continuous Classification Scheme for Multifunction Myoelectric Control," IEEE Trans. Biomed. Eng., vol. 48, pp.302-311, 2001.

[6] M. Zecca, S. Micera, M. C. Carrozza and P. Dario, "Control of Multifunctional Prosthetic Hands by Processing the Electromyographic Signal," Crit. Rev. Biomed. Eng., vol. 30, pp. 459-485, 2002.

[7] B. Karlik, M. O. Tokhi and M. Alci, "A Fuzzy Clustering Neural Network Architecture for Multifunction Upper-Limb Prosthesis," IEEE Trans. Biomed. Eng., vol. 50, pp.12551261, 2003.

[8] P. Shenoy, K. J. Miller, B. Crawford and R. P. Rao, "Online Electromyographic Control of a Robotic Prosthesis," IEEE Trans. Biomed. Eng., vol. 55, pp. 1128-1135, 2008.

[9] G. Li, A. E. Schultz and T A. Kuiken, "Quantifying Patter Recognition-Based Myoelectric Control of Multifuntional Transradial Prostheses," IEEE Trans. Biomed. Eng., vol. 18, pp. 185-192, 2010.

[10] A. Andrews, E. Morin and L. McLean, "Optimal Electrode Configurations for Finger Movement Classification using EMG," Proc. 31st Ann. Int. Conf. IEEE EMBS, 2009, pp. 2987-2990.

[11] D. Peleg, E. Braiman, E. Yom-Tov and G. F. Inbar, "Classification of Finger Activation for Use in a Robotic Prosthesis Arm," IEEE Trans. Neural Sys. Rehab. Eng., vol. 10. pp. 290-293, 2002.

[12] J. Z. Wang, R. C. Wang, F. Li, W. Jiang and D. W. Jin, "EMG Signal Classification for Myoelectric Teloperating a Dexterous Robot Hand," Proc. 27th Ann. Int. Conf. IEEE EMBS, 2005, pp. 5931-5933.

[13] F. V. G. Tenore, A. Ramos, A. Fahmy, S. Acharya, R. Etienne-Cummings and N.V. Thakor, "Decoding of Individuated Finger Movements Using Surface Electromyography," IEEE Trans. Biomed. Eng., vol. 56, pp. 1427-1434, 2009.

[14] C. Castellini and P. van der Smagt, "Surface EMG in Advanced Hand Prosthetics," Bio. Cyber., vol. 100, pp. 3547, 2009.

[15] R. J. Smith, F. Tenore, D. Huberdeau, R. Etienne-Cummings and N. V. Thakor, "Continuous Decoding of Finger Position from Surface EMG Signals for the Control of Powered Prostheses," Proc 30th Ann. Int. Conf. IEEE EMBS, 2008, pp. 197-200.

[16] R. J. Smith, D. Huberdeau, F. Tenore and N. V. Thakor, "Real-Time Myoelectric Decoding of Individual Finger
Movements For a Virtual Target Task," Proc. 31st Ann. Int. Conf. IEEE EMBS, 2009, pp. 2376-2379.

[17] S. Muceli and D. Farina, "Simultaneous and proportional estimation of hand kinematics from EMG during mirrored movements at multiple degrees-of-freedom, "IEEE Trans. Neural Sys. Rehabil. Eng., vol. 20, pp. 371-378, 2012.

[18] H. Reucher, G. Rau and J. Silny, "Spatial Filtering of Noninvasive Multielectrode EMG. I: Introduction to Measuring Technique and Implications," IEEE Trans. Biomed. Eng., vol. 34, pp. 98-105, 1987.

[19] H. Reucher, J. Silny and G. Rau, "Spatial Filtering of Noninvasive Multielectrode EMG. II: Filter performance in theory and modeling," IEEE Trans. Biomed. Eng., vol. 34, pp. 106-113, 1987.

[20] C. Disselhorst-Klug, J. Bahm, V. Ramaekers, A. Trachterna and G. Rau, "Non-invasive approach of motor unit recording during muscle contractions in humans," Eur. J. Appl. Physiol., vol. 83, pp. 144-150, 2000.

[21] A. O. Perotto, Anatomical Guide for the Electromyographer. Third edition, Springfield, IL: Charles C Thomas, 1994, pp. 30-73.

[22] L. Ljung, System Identification: Theory for the User. Upper Saddle River, NJ: Prentice-Hall, 1999, pp. 491-519.

[23] E. A. Clancy, O. Bida, and D. Rancourt, "Influence of advanced electromyogram (EMG) amplitude processors on EMG-to-torque estimation during constant-posture, forcevarying contractions," J. Biomech., vol. 39, pp. 2690-2698, 2006.

[24] E. A. Clancy, L. Liu, P. Liu and D. V. Z. Moyer, "Identification of constant-posture EMG-torque relationship about the elbow using nonlinear dynamic models," IEEE Trans. Biomed. Eng., vol. 59, pp. 205-212, 2012.

[25] D. Farina, C. Fevotte, C. Doncarli and R. Merletti, "Blind separation of linear instantaneous mixtures of nonstationary surface myoelectric signals," IEEE Trans. Biomed. Eng., vol. 51, pp. 1555-1567, 2004. 